\title{
Lactobacillus acidophilus regulates STAT3 and STAT5 signaling in bovine $\beta$-lg-sensitized mice model
}

\author{
Yun Zhang ${ }^{1} \cdot$ Ai-li $\mathrm{Li}^{1} \cdot$ Yi-qiao Sun ${ }^{1} \cdot$ Peng Du ${ }^{1}$ • \\ Li-bo Liu ${ }^{1}$ - Shuang $\mathrm{Li}^{1}$. Chao Zhang ${ }^{1}$
}

Received: 13 October 2015 / Revised: 12 January 2016 / Accepted: 26 February 2016 /

Published online: 23 March 2016

(C) INRA and Springer-Verlag France 2016

\begin{abstract}
Our previous study has shown that oral supplementation with Lactobacillus acidophilus KLDS 1.0738 could inhibit $\beta$-lactoglobulin $(\beta-\lg )$ allergy. In this study, we investigated the effect of $L$. acidophilus on the balance between T helper type 17 (Th17) cells and regulatory T cells (Treg) in allergic mouse model and explored the participation of related signal transducers and activator of transcription (STAT) in this process. Bovine $\beta$-lg-sensitized mice received strains KLDS 1.0738 for 3 weeks. After the allergen challenge, the percentages of Treg and Th17 cells, cytokine and STAT mRNA expression, and pSTAT protein levels were detected by flow cytometry, quantitative RT-PCR, and western blot, respectively. The results showed that stimulation with $\beta-\lg$ increased the levels of IL-6, pSTAT3, and Th17 cells, but decreased the levels of IL-2, pSTAT5, and Treg cells compared to the controls $(P<0.05)$. However, oral administration of $L$. acidophilus KLDS 1.0738 suppressed $\beta$-lg-induced inflammatory and improved the Treg/Th17 imbalance. In addition, L. acidophilus-treated group presented decrease in pSTAT3 activation, SOCS3, and IL-6 level, but increase in STAT5a/b, CD25, and IL-2 mRNA expression. These findings suggest that L. acidophilus could regulate IL-6/STAT3 and IL-2/STAT5 pathway, which may be responsible for the Treg/Th17 imbalance in $\beta$-lg-sensitized mice.
\end{abstract}

Keywords Lactobacillus acidophilus · Milk allergy · Treg/Th17 imbalance · STAT3/ STAT5 signaling pathway

Ai-li Li

aili-mail@163.com

1 Key laboratory of Dairy Science, Ministry of Education, College of Food Science, Northeast Agriculture University, No.59, Mucai Street, Xiangfang District, Harbin, Heilongjiang 150030, China 


\section{Introduction}

Cow's milk allergy (CMA) is a complex inflammatory disease in which CD4 ${ }^{+}$ $\mathrm{T}$ helper (Th) cells play a central role (Giovanna et al. 2012). In addition to the general paradigm of Th1/Th2 immune regulation, the current study showed that two additional Th cell subsets, interleukin 17 (IL-17)-producing Th17 cells and regulatory $\mathrm{T}$ cells (Treg), were also partially responsible for the development of allergic inflammation (Palomares et al. 2010; Zhao et al. 2010). Therefore, a better understanding of the roles of Treg and Th17 cells may provide insights into effective CMA control.

Most evidence demonstrated that the STAT pathways were involved in Th cell differentiation (Saleh et al. 2009; Chen et al. 2007) and correlated with the inflammatory process (Minegishi et al. 2007). Among the STAT family of proteins, STAT3 was reported to promote Th17 differentiation, including the induction of Th17-related cytokine production, ROR $\gamma \mathrm{t}$ and $\operatorname{ROR} \alpha$ (Harris et al. 2007). In addition, STAT3 might be activated by most proinflammatory agents in the proallergic milieu, such as IL-6 (Yang et al. 2007). Mori et al. (2011) showed that the IL-6/STAT3 pathway was critical in chronic intestinal inflammation by facilitating Th17 cells and restraining Treg. In contrast, STAT5 was activated by IL-2 and was indispensable in maintaining Treg homeostasis and self-tolerance (Laurence et al. 2007; Yao et al. 2006). Burchill et al. (2007) indicated that STAT5a/b promoted Treg development and maintenance through regulation of both forkhead box P3 (Foxp3) and CD25 expression. More recently, Wang et al. (2012) suggested that the Th17/Treg imbalance caused by nasal polyposis was positively correlated with STAT3/STAT5 activation. However, it is not yet known whether IL-6/STAT3 and IL-2/STAT5 pathways might contribute to the Th17/Treg imbalance in CMA.

Lactobacilli have beneficial effects on T-cell-mediated inflammatory diseases. Previous research reported that probiotics could prevent food allergy by improving the Th1/Th2 imbalance (Kim et al. 2008). Recent studies indicated that application of lactobacilli could attenuate airway hyperreactivity by induction of Tregs in a mouse model of asthma (Jan et al. 2012; Karimi et al. 2009). We also showed Lactobacillus acidophilus strain KLDS 1.0738 had the ability to suppress the $\beta-\lg$ allergic symptoms and Th17 cytokine production ( $\mathrm{Li}$ et al. 2013). Moreover, some studies focused on the effect of probiotics on activating the cytokine-mediated STAT pathways to regulate the immune responses. Miettinen et al. (2000) reported that L. rhamnosus GG ATCC 53103 could induce Th1 cytokine production through the NF-kB and STAT pathways in the macrophages. Jandu et al. (2009) found that L. helveticus prevented IFN- $\gamma-J a k 1$ and 2-STAT-1 activation in Escherichia coli O157:H7-infected cells. However, whether lactobacilli might modulate STAT signaling in response to the Th17/Treg development in $\beta$-lg-sensitized mice is currently unknown. Therefore, the aim of this research is to evaluate the relationship between STAT pathways and the imbalance of Treg/Th17 in $\beta-\lg$ sensitized mice and to analyze the effect of L. acidophilus on the activations of STAT3 and STAT5. 


\section{Materials and methods}

\subsection{Mice and bacteria}

Six-week-old BALB/c mice were purchased from the Harbin Veterinary Research Institute (Harbin, China) and raised in normal husbandry environment. The mice were fed a milk-free standard diet and provided with water ad libitum. All animal experiments complied with the rules of the Care and Use of Laboratory Animals of Northeast Agricultural University.

The L. acidophilus KLDS 1.0738 used in this study was obtained from the Key Laboratory of Dairy Science, Ministry of Education (Northeast Agriculture University, China). The bacteria were incubated at $37{ }^{\circ} \mathrm{C}$ in Man-Rogosa-Sharpe broth until they reached a concentration of $5 \times 10^{9} \mathrm{CFU} / \mathrm{mL}$. The bacteria cells were collected by centrifugation (4000 g, $10 \mathrm{~min}$ ), washed three times with sterile distilled water, and heat-killed at $100{ }^{\circ} \mathrm{C}$ for $20 \mathrm{~min}$. Then, the treated organisms were lyophilized and suspended in $0.9 \%$ sterile saline.

\subsection{Experimental groups}

Animal experiments were carried out as described previously with minor modifications (Li et al. 2013). As shown in Fig. 1, BALB/c mice were randomly divided into three groups: $\beta$-lg allergy mice $(\beta$-Lg) were sensitized by intraperitoneal injection of $50 \mu \mathrm{g}$ $\beta$-lg (Sigma-Aldrich, USA) adsorbed on $2 \mathrm{mg}$ aluminum hydroxide (Sigma) at days 7 , 14 , and 21 ; L. acidophilus-treated mice (LR) were intragastrically administered with $200 \mu \mathrm{L}$ of L. acidophilus suspension ( $2.5 \mathrm{mg}$ /animal) three times a week from days 1 to 21 ; control mice (Con) were treated with $0.9 \%$ sterile saline. On day 25 , the mice were orally challenged twice with $20 \mathrm{mg} \beta-\lg$ or saline solution, and then all the animals were sacrificed in $2 \mathrm{~h}$ after the last $\beta$-lg challenge. The blood, lung, colon, and spleen tissues were subsequently collected.

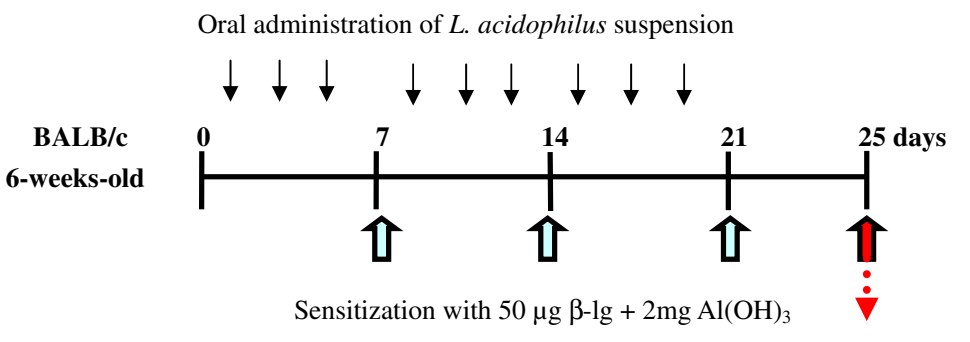

Oral challenge

Fig. 1 Schedule for immunization with $\beta-\lg$ and oral administration of Lactobacillus acidophilus KLDS 1.0738. Mice were sensitized by intraperitoneal injection of with $\beta$-lg once a week for 3 weeks. L. acidophilus KLDS 1.0738 (dose of $2.5 \mathrm{mg} /$ mouse) was administered orally three times a week from days 1 to 21 . As a control, $200 \mu \mathrm{L}$ of distilled water was administered. Oral challenge with and without $\beta$-lg was carried out on day 25 


\subsection{Eosinophil counts}

The blood samples were collected and the plasma was obtained. The cell counts were performed using a hemacytometer under light microscope after staining with eosinacetone diluent.

\subsection{Histological analysis of the colon and lung tissues}

The colon and lung tissues were removed and fixed in $10 \%$ formalin, and then the specimens were dehydrated and embedded in paraffin. The paraffin sections were stained with hematoxylin and eosin (HE). Pathological alterations in the lung and colon tissues were assessed under a light microscope.

\subsection{Flow cytometric analysis}

Single-cell suspensions were prepared from spleens by injecting PBS into the tissues, and the erythrocytes were lysed before the samples were subjected to flow cytometric analysis. To detect the Treg, FITC-labeled anti-CD4 and PE-labeled anti-CD25 (eBioscience, USA) were used for cell surface staining, and APC-labeled anti-Foxp3 (eBioscience, USA) was applied for intracellular staining after fixation and permeabilization. To detect the Th17 cells, the cells were first surface-stained with FITCconjugated CD4 Abs, then fixed and permeabilized according to the kit manual, and finally stained intracellularly with APC-conjugated IL-17A Abs. After washing three times with PBS, the cells were resuspended and processed with a FACS flow cytometer equipped with Cell Quest software (BD FACS Aria ${ }^{\mathrm{TM}}$ Cell Sorter, USA).

\subsection{RNA isolation and real-time PCR}

Total RNA was isolated from the spleen tissues using the RNA simple Total RNA kit (Tiangen, China), and cDNA was synthesized using the cDNA RT reagent kit (Takara, China). The STAT3, STAT5a/b, SOCS3, CD25, IL-2, and IL-6 message expression levels were quantified using the ABI 7500 Real-Time PCR System (Applied Bio systems, USA). Amplification was performed in a total volume of $25 \mu \mathrm{L}$ for 40 cycles, and the products were detected using SYBR Premix Ex Taq ${ }^{\mathrm{TM}}$ II (Takara, China). The sequences of the specific primers used in the PCR are shown in Table 1 and the mRNA expression in each group were normalized to the level of $\beta$-actin housekeeping genes using the $2^{-\triangle \Delta \mathrm{Ct}}$ method and represented as the fold induction.

\subsection{Extraction of the tissue protein and western blot analysis}

Proteins from the CD4 T cell fraction were extracted with RIPA lysis buffer (Solarbio, China) and the concentration was detected using BCA kit (Solarbio, China) following the manufacturer's protocol. The samples containing $25 \mu \mathrm{g}$ of protein were boiled, separated by polyacrylamide gel electrophoresis, and transferred to a polyvinylidene fluoride membrane. The membrane was allowed to react with polyclonal anti-GAPDH, rabbit anti-STAT3, anti-phospho-STAT3 (Tyr), anti-STAT5, and anti-phospho-STAT5 (Tyr) (Cell Signaling, USA). After incubation with a horseradish peroxidase (HRP)- 
Table 1 Primers used for real-time PCR

\begin{tabular}{|c|c|c|}
\hline Name & Sequence $\left(5^{\prime} \rightarrow 3^{\prime}\right)$ & \\
\hline \multirow[t]{2}{*}{ STAT5a } & Forward primer & AAGATCAAGCTGGGGCACTA \\
\hline & Reverse primer & ATGGGACAGCGGTCATAC \\
\hline \multirow[t]{2}{*}{ STAT5b } & Forward primer & CGAGCTGGTCTTTCAAGTCA \\
\hline & Reverse primer & CTGGCTGCCGTGAACAAT \\
\hline \multirow[t]{2}{*}{ STAT3 } & Forward primer & CAAAACCTCAAGAAGCCAAGG \\
\hline & Reverse primer & TCACTCACAATGCTTCTCCGC \\
\hline \multirow[t]{2}{*}{$\mathrm{CD} 25$} & Forward primer & ACACCTGTAAGCCCAGCTCT \\
\hline & Reverse primer & TGGAAAGGTTGAGGGGTAAG \\
\hline \multirow[t]{2}{*}{ SOCS3 } & Forward primer & GAAGACCAAGTTCATCTGTGTG \\
\hline & Reverse primer & GTAGCACACTCCGAGGTCAGAT \\
\hline \multirow[t]{2}{*}{ IL-6 } & Forward primer & GATGCTACCAAACTGGATATAATC \\
\hline & Reverse primer & GGTCCTTAGCCACTCCTTCTGTG \\
\hline \multirow[t]{2}{*}{ IL-2 } & Forward primer & CACATTTGAGTGCCAATTCGAT \\
\hline & Reverse primer & GCGCTTACTTTGTGCTGTCCTA \\
\hline \multirow[t]{2}{*}{$\beta$-actin } & Forward primer & CGCAAAGACCTGTATGCCAAT \\
\hline & Reverse primer & GGGCTGTGATCTCCTTCTGC \\
\hline
\end{tabular}

conjugated anti-rabbit antibody, the membrane was incubated with ECL chemiluminescence reagent (TransGen Biotech, China), and the film was exposed to the membrane.

\subsection{Statistical analysis}

All data in the text and figures were presented as the means \pm standard deviation (means $\pm \mathrm{SD}$ ). One-way ANOVA was performed for detection of significant differences among the groups. The statistical analysis was carried out using the multiple comparison test (SPSS17.0 software). $P$ values $<0.05$ were considered significantly different.

\section{Results}

\subsection{Effect of $L$. acidophilus on eosinophil counts and histologic characteristics of $\beta$-lg-sensitized mice}

As shown in Fig. 2a, $\beta$-lg allergen caused eosinophil-rich inflammation in allergic mice. In contrast, treatment with $L$. acidophilus decreased the percentages of eosinophils in the blood of sensitized mice.

The severity of allergy symptoms was further investigated by means of histopathology. Bovine $\beta$-lg challenge led to inflammatory cell infiltrates into the lung and the colon tissues of sensitized mice. However, decreased inflammatory responses were observed in L. acidophilus-treated group (Fig. 2b, c). 
A

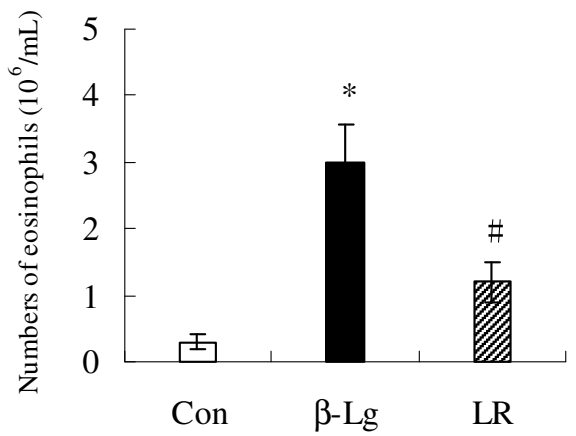

\section{B}
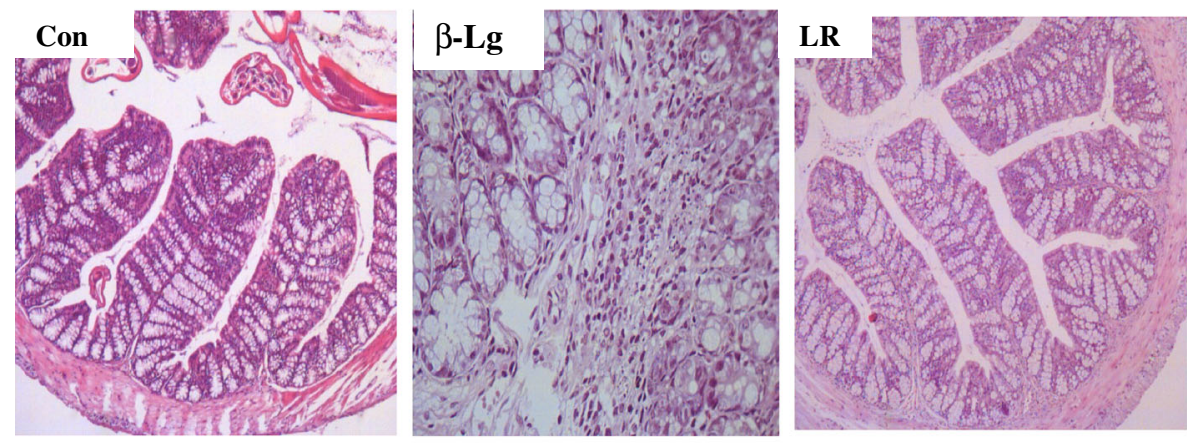

C

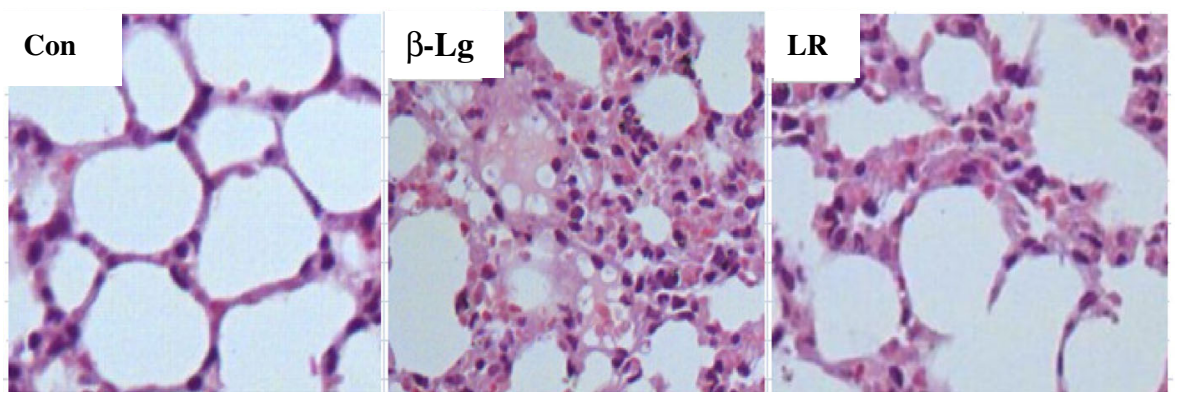

Fig. 2 Effect of L. acidophilus treatment on the eosinophil counts, colon, and lung architecture in $\beta$-lg allergy mice. Mice were sensitized with $\beta-\lg (\beta-\mathrm{Lg})$ on days 7,14 , and 21. L. acidophilus was given orally to the mice (LR) from day 1 to day 21. After antigen challenge, blood samples were prepared and the eosinophil counts were performed (a). Histological sections of murine colons (b) and lung tissues (c) were stained with HE ( $\times 400$ magnification). The data are the means \pm S.D. $(n=6) ; * P<0.05$ versus control group, ${ }^{\#} P<0.05$ versus $\beta-\lg$ group

\subsection{Effect of $L$. acidophilus on the Th17/Treg imbalance in $\beta$-lg-sensitized mice}

In agreement with our previous study, the $\beta$-lg challenge caused the frequencies of the Th17 cells $\left(\mathrm{CD} 4^{+} \mathrm{IL}-17^{+} / \mathrm{CD}^{+} \mathrm{T}\right.$ cells) to increase and the frequencies of the Treg $\left(\mathrm{CD} 4^{+} \mathrm{CD} 25^{+} \mathrm{Foxp}^{+} \mathrm{T}\right.$ cells) to decrease in the allergic mice compared to the controls 
$(P<0.05)$. Subsequently, the effect of $L$. acidophilus on regulating the Treg/Th17 cell differentiation was assessed. As shown in Fig. 3, in L. acidophilus-treated mice, the numbers of Th17 in $\mathrm{CD}^{+}$splenocytes were lower and the numbers of Treg cells were higher than those of $\beta$-lg-sensitized mice $(P<0.05)$. Furthermore, the Th17/Treg ratio was increased in the spleens of allergic mice compared with the controls, whereas the Th17/Treg ratio of the L. acidophilus-treated group was reduced to $0.84 \pm 0.27$.

\subsection{Effect of $L$. acidophilus on signal transduction in $\beta$-lg-sensitized mice}

We next detected the mRNA expression of correlative signaling caused by the Treg/Th17 responses in the spleens of mice from each group. As shown in Fig. 4, the splenic STAT3, SOCS3, and IL-6 mRNA levels were increased, but the STAT5,
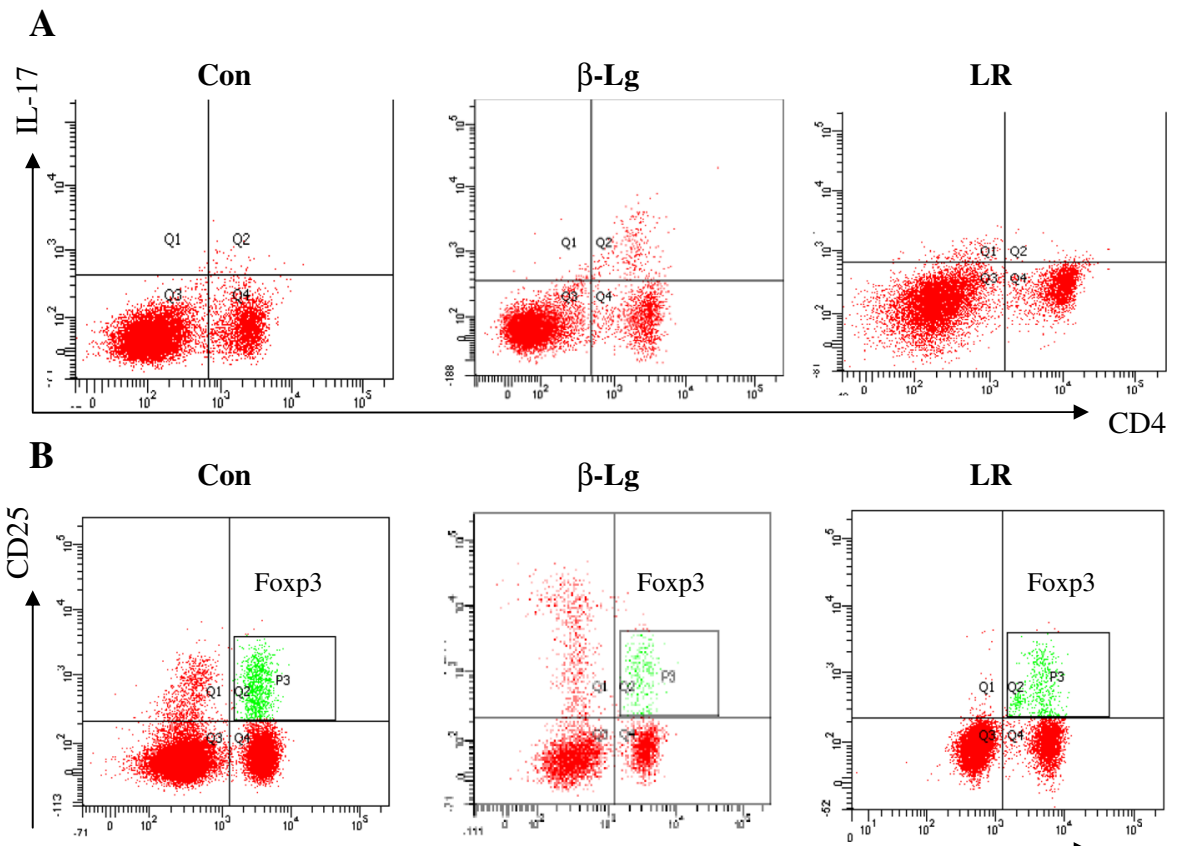

\section{$\mathbf{L R}$}

C
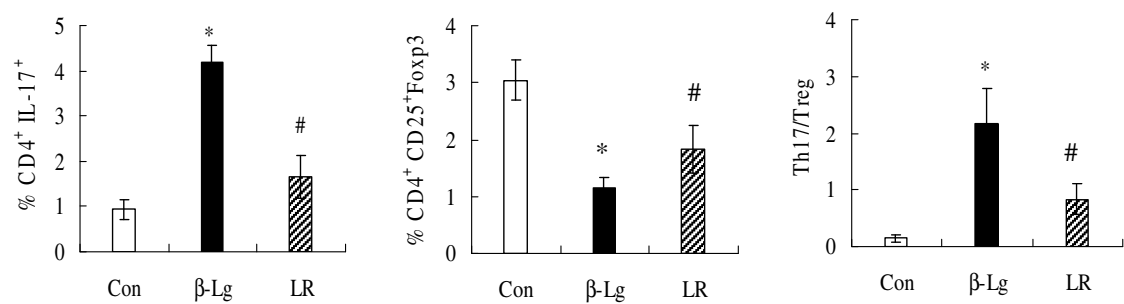

Fig. 3 Effect of L. acidophilus administration on the Treg and Th17 cell populations in $\beta$-lg-induced allergic mice. The splenocyte $\mathrm{CD}^{+}$cells from each group were stained by surface antibodies followed by intracellular antibodies as described in methods. a The percentages of Th17 in CD4 ${ }^{+}$cells in the spleens; $\mathbf{b}$ the percentages of Tregs in the $\mathrm{CD}^{+}$cells in the spleens; $\mathbf{c}$ the ratio of Th17 cells to Treg cells. The data are the means \pm S.D. $(\mathrm{n}=6) ;{ }^{*} P<0.05$ versus control group, ${ }^{\#} P<0.05$ versus $\beta$-lg group 
A
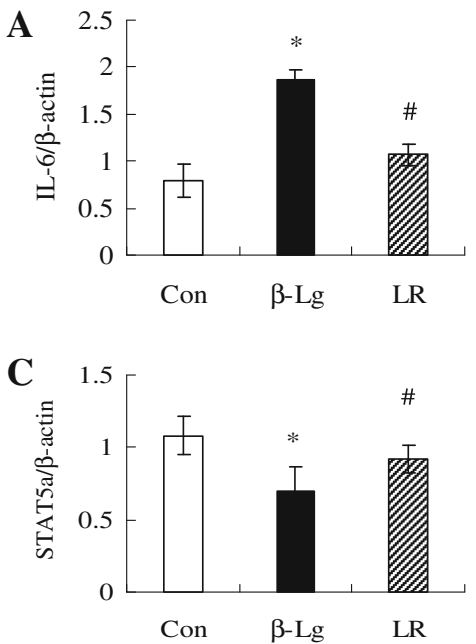

$\mathbf{E}$

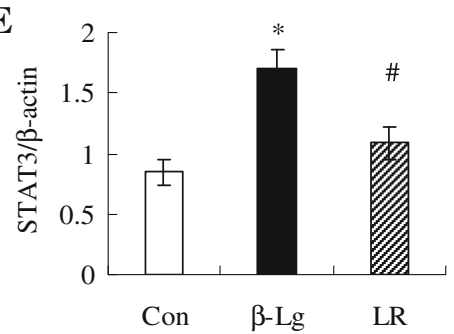

G

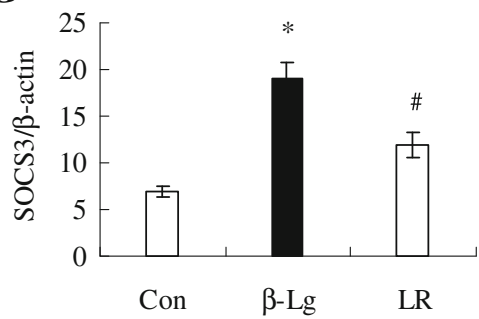

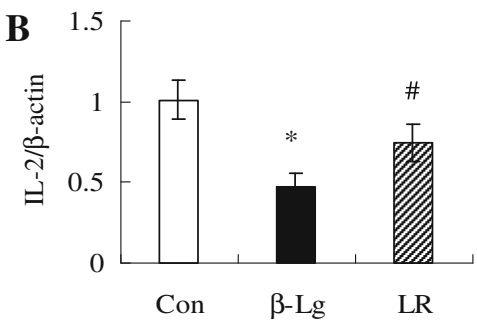

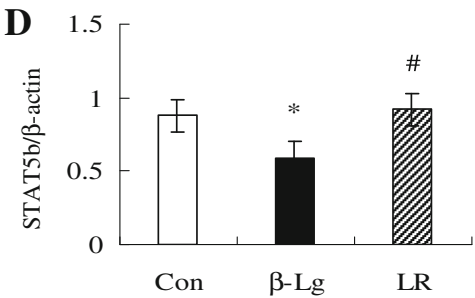

$\mathbf{F}$

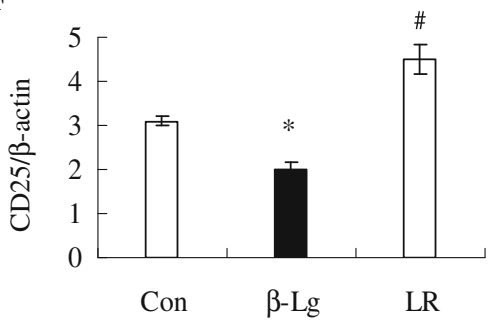

Fig. 4 Effect of $L$. acidophilus on related cytokine and STAT signal mRNA expression in the spleen of $\beta$-lgsensitized mice. The relative levels of a IL-6, b IL-2, c STAT5a, d STAT5b, e STAT3, f CD25, and g SOCS3 mRNA in the spleens of mice from each group were calculated and expressed at the percentage of mRNA to $\beta$-actin. The data are means \pm S.D. $(n=6) ;{ }^{*} P<0.05$ versus control group, ${ }^{\sharp} P<0.05$ versus $\beta$-lg group

CD25, and IL-2 mRNA levels were decreased in the $\beta$-lg allergy group compared to the controls $(P<0.05)$. However, oral consumption of L acidophilus showed a tendency to inhibit the upregulation of STAT3 and IL-6 mRNA expression and prevent the downregulation of STAT5a/b and IL-2 levels in sensitized mice.

\subsection{Effect of $L$. acidophilus on the STAT pathways in the sensitized mice}

First, $\beta$-lg challenge induced a transient phosphorylation of STAT3 in the spleen tissues of the sensitized mice (Fig. 5). The time-course examination revealed STAT3 
A

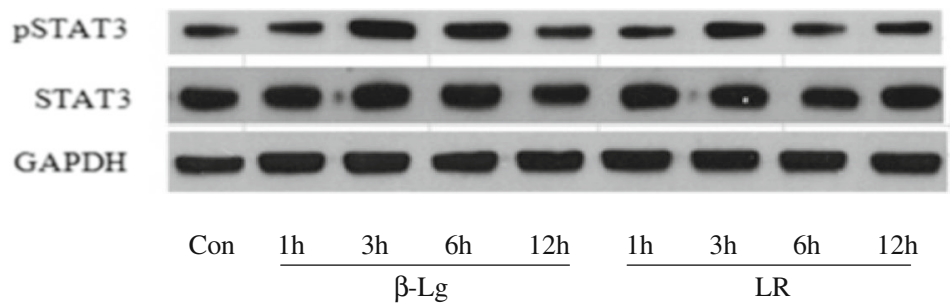

B

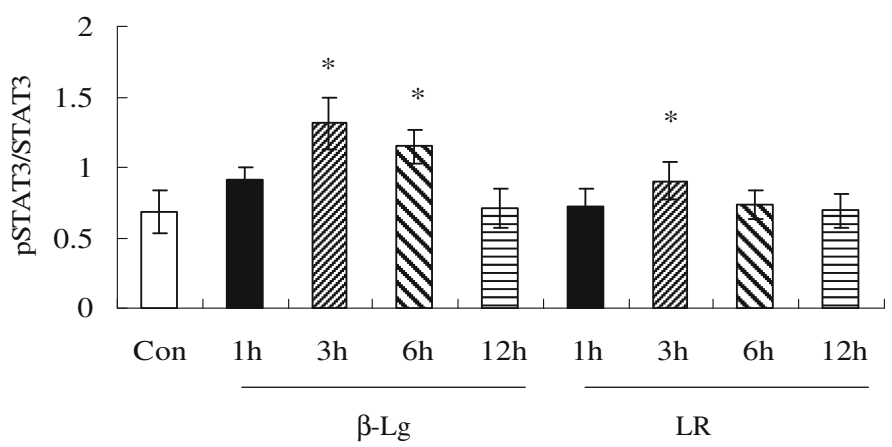

Fig. 5 A time-course change of pSTAT3 level in mouse spleen tissues. After antigen challenge, the pSTAT3, STAT3, and GAPDH protein samples from the spleens at different time points $(1,3,6$, and $12 \mathrm{~h})$ were subjected to western blot analyses. a The conventional western blots of pSTAT3 (upper), total STAT3 (middle), and GAPDH (lower); $\mathbf{b}$ the expression of pSTAT3/STAT3. Data are the means \pm S.D. $(n=6)$; $* P<0.05$ versus control group

phosphorylation was increased at 3-6 h and returned to the baseline at $12 \mathrm{~h}$. At the same time, the levels of pSTAT3 were lower in L. acidophilus-treated group. On the other hand, the phosphorylation of STAT5 was decreased at 3-6 h after oral antigen challenge (Fig. 6). Meanwhile, the pSTAT5 in the spleen tissues of L. acidophilustreated group was increased compared to allergy group $(P<0.05)$.

\section{Discussion}

Recently, the Treg and Th17 cells were shown to be associated with the initiation and perpetuation of inflammation (Bettelli et al. 2006; Cheng et al. 2008; Xie et al. 2010). In previous study, we found the Th17/Treg imbalance was involved in $\beta$-lg allergy, characterized by proliferation of inflammatory cells and predominantly Th17 response in sensitized mice (Li et al. 2014). Recently, some studies speculated that activation of STAT3 and STAT5 might be closely related to the generation of the Treg and Th17 cells and the pathogenesis of allergic disease. Paul et al. (2009) reported the role of STAT3, IL-6, and SOCS3 in the ovalbumin-induced mouse model of asthma. Wei et al. (2008) revealed that STAT3 was indispensable in Th17 cell maintenance. In this study, we further found the challenge with $\beta-\mathrm{LG}$ induced a time-dependent increase in STAT3 phosphorylation, as well as an increase in IL-6, SOCS3, and STAT3 mRNA expression levels. Those may be the signal leading to Th17 differentiation in $\beta$-lg-sensitized mice. Furthermore, the transient decrease of phosphorylated STAT5 and lower CD25 and IL2 mRNA levels also occurred in the spleens of the allergic mice. Laurence et al. (2007) 
A

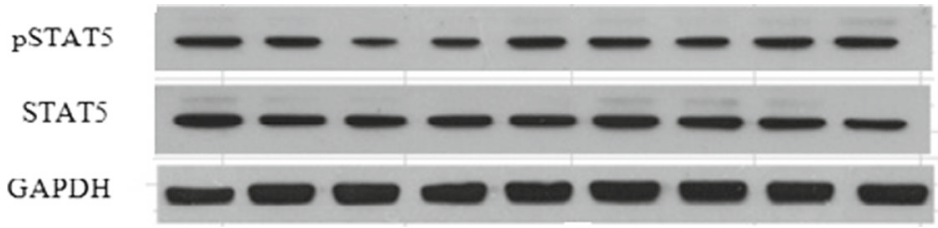

Con $\frac{1 \mathrm{~h} \quad 3 \mathrm{~h} \quad 6 \mathrm{~h} \quad 12 \mathrm{~h}}{\beta-\mathrm{Lg}} \quad \frac{1 \mathrm{~h} \quad 3 \mathrm{~h} \quad 6 \mathrm{~h} \quad 12 \mathrm{~h}}{\mathrm{LR}}$

B

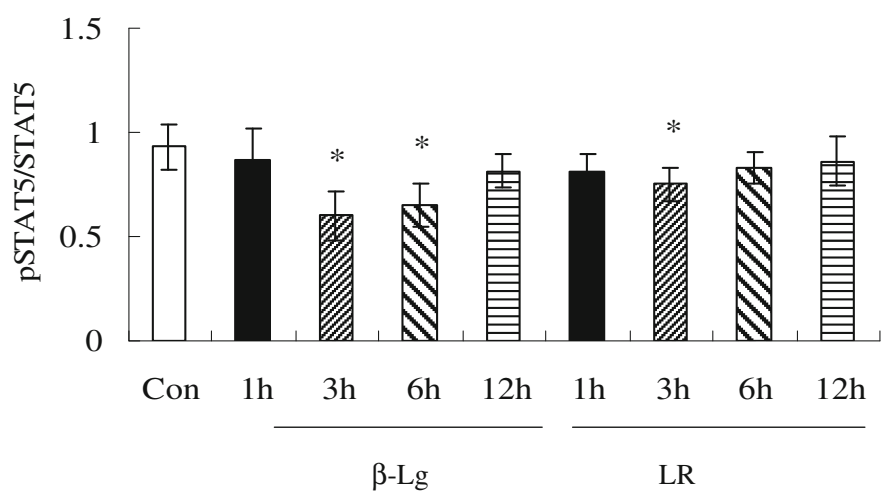

Fig. 6 A time-course change of pSTAT5 level in mouse spleen tissues. After antigen challenge, the pSTAT5, STAT5, and GAPDH protein expression profiles at different time points $(1,3,6$, and $12 \mathrm{~h}$ ) were subjected to western blot analyses. a Representative western blots of pSTAT5 (upper), total STAT5 (middle), and GAPDH (lower). b The levels of pSTAT5/STAT5. Data are the means \pm S.D. $(n=6) ; * P<0.05$ versus control group

provided evidence that IL-2 and STAT5a/b were the key regulators of Treg and served to constrain Th17 polarization. Yang et al. (2011) reported that the induction of STAT5 binding by IL-2 was beneficial to STAT3 suppression. Thus, this study suggests that the activation of IL-6/STAT3 pathway might be involved in the progression of $\beta$-lg allergy. In addition, the diminishing of IL-2/STAT5 pathway might further inhibit Treg differentiation but promote Th17 development in allergic mice.

Subsequently, we observed that oral administration of L. acidophilus KLDS 1.0738 suppressed $\beta$-lg-induced inflammatory, including alleviating eosinophil inflammatory influx and the severe histologic features in the colons and lungs of the sensitized mice. It was previously shown that $L$. acidophilus treatment could increase the Treg-related cytokines and transcription factor production (IL-10, TGF- $\beta 1$, Foxp3) but suppress the Th17-type levels (IL-17A, ROR $\gamma$ t) (Li et al. 2013). In this study, addition of L. acidophilus to $\beta$-lg-immunized mice could significantly upregulate the number of Treg cells and downregulate the number of Th17 cells compared with the allergy group $(P<0.05)$. These results supported that the anti-allergy effects of $L$. acidophilus were associated with a reversal of the Th17/Treg imbalance toward the Treg dominance. Some research showed that JAK-STAT signaling pathway modulation by probiotic might have important effects on alleviating the severity of inflammatory. For instance, Sun et al.(2005) indicated that the expression of IL-12 and STAT 4 could be greatly increased by the peptidoglycan derived from Lactobacillus sp. Lee et al. (2010) demonstrated that the probiotic administration-induced SOCS expression through the 
STAT1/STAT3 pathway could inhibit Helicobacter pylori infection. This study showed that application of L. acidophilus led to enhancement of CD25 and IL-2 mRNA expressions, accompanied with upregulation of STAT5 mRNA. In contrast, IL-6 and STAT3 expression in L. acidophilus-treated group were lower than those in allergy group. Similar to the results of mRNA trends, the phosphorylation of both STAT3 and STAT5 transiently occurred in response to the distinct cytokines after L. acidophilus stimulation. Low levels of pSTAT3 and high levels of pSTAT5 were consistent with increased Treg numbers and decreased Th17/IL-17 numbers in L. acidophilus-treated group. Therefore, IL-2/STAT5 pathway induced by L. acidophilus may be a potent negative regulator of Th17 differentiation in CMA.

In conclusion, the increase of the IL-6/STAT3 pathway may contribute to the Th17/Treg imbalance caused by $\beta$-lg allergy. The Treg-dominant immunity stimulated by L. acidophilus may be positively correlated to the IL-2/STAT5 pathway. However, this study was limited because the JAK-STAT pathways served as a major cytokine signaling pathway which was involved in many biological processes. Therefore, further studies are recommended to explore the anti-inflammatory mechanism of L. acidophilus, which will be helpful in application of probiotics to prevent various allergies.

Acknowledgments This work was financially supported by the Education Department of Heilongjiang Province backbone teachers (1254G008) and the Natural Science Foundation of Heilongjiang Province (C201423).

\section{References}

Bettelli E, Carrier Y, Gao W, Korn T, Strom TB, Oukka M, Weiner HL, Kuchroo VK (2006) Reciprocal developmental pathways for the generation of pathogenic effector $T_{H} 17$ and regulatory $T$ cells. Nature 441(7090):235-238

Burchill MA, Yang J, Vogtenhuber C, Blazar BR, Farrar MA (2007) IL-2 receptor $\beta$-dependent STAT5 activation is required for the development of Foxp $3^{+}$regulatory T cells. J Immunol 178(1):280-290

Chen Z, Laurence A, O'Shea JJ (2007) Signal transduction pathways and transcriptional regulation in the control of Th17 differentiation. Semin Immunol 19(6):400-408

Cheng X, Yu X, Ding YJ, Fu QQ, Xie JJ, Tang TT, Yao R, Chen Y, Liao YH (2008) The Th17/Treg imbalance in patients with acute coronary syndrome. Clin Immunol 127(1):89-97

Giovanna V, Carla C, Alfina C, Domenico PA, Elena L (2012) The immunopathogenesis of cow's milk protein allergy (CMPA). Ital J Pediatr 38:35

Harris TJ, Grosso JF, Yen HR, Xin H, Kortylewski M, Albesiano E, Hipkiss EL, Getnet D, Goldberg MV, Maris CH, Housseau F, Yu H, Pardoll DM, Drake CG (2007) Cutting edge: an in vivo requirement for STAT3 signaling in $\mathrm{T}_{\mathrm{H}} 17$ development and $\mathrm{T}_{\mathrm{H}}$ 17-dependent autoimmunity. J Immunol 179(7):43134317

Jan RL, Yeh KC, Hsieh MH, Lin YL, Kao HF, Li PH, Chang YS, Wang JY (2012) Lactobacillus gasseri suppresses Th17 pro-inflammatory response and attenuates allergen-induced airway inflammation in a mouse model of allergic asthma. Brit J Nutr 108(1):130-139

Jandu N, Zeng ZJ, Johnson-Henry KC, Sherman PM (2009) Probiotics prevent enterohaemorrhagic Escherichia coli $\mathrm{O} 157: \mathrm{H} 7-$ mediated inhibition of interferon-gamma- induced tyrosine phosphorylation of STAT-1. J Microbiol 155(Pt 2):531-540

Karimi K, Inman MD, Bienenstock J, Forsythe P (2009) Lactobacillus reuteri-induced regulatory T cells protect against an allergic airway response in mice. Am J Resp Crit Care 179(3):186-193

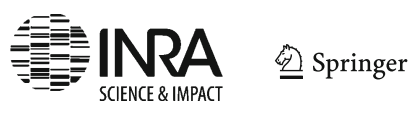


Kim JY, Choi YO, Ji GE (2008) Effect of oral probiotics (Bifidobacterium lactis AD011 and Lactobacillus acidophilus AD031) administration on ovalbumin-induced food allergy mouse model. J Microbiol Biotechnol 18(8):1393-1400

Laurence A, Tato CM, Davidson TS, Kanno Y, Chen Z, Yao Z, Blank RB, Meylan F, Siegel R, Hennighausen L, Shevach EM, O'Shea JJ (2007) Interleukin-2 signaling via STAT5 constrains T helper 17 cell generation. Immunity 26(3):371-381

Lee JS, Paek NS, Kwon OS, Hahm KB (2010) Anti-inflammatory actions of probiotics through activating suppressor of cytokine signaling (SOCS) expression and signaling in helicobacter pylori infection: a novel mechanism. J Gastroen Hepatol 25(1):194-202

Li AL, Meng XC, Duan CC, Huo GC, Zheng QL, Li D (2013) Suppressive effects of oral administration of heat-killed Lactobacillus acidophilus on Th17 immune responses in a bovine $\beta$-lg-sensitized mice model. Biol Pharm Bull 36(2):202-207

Li AL, Meng XC, Huo GC, Duan CC, Huo GC, Zheng QL, Li D (2014) The Treg/Th17 imbalance in bovine $\beta-\lg -$ sensitized mice. Int Dairy J 34(2):257-262

Miettinen M, Lehtonen A, Julkunen I, Matikainen S (2000) Lactobacilli and streptococci activate NF-kappa B and STAT signaling pathways in human macrophages. J Immunol 164(7):3733-3740

Minegishi Y, Saito M, Tsuchiya S, Tsuge I, Takada H, Hara T, Kawamura N, Ariga T, Pasic S, Stojkovic O, Metin A, Karasuyama H (2007) Dominant-negative mutations in the DNA- binding domain of STAT3 cause hyper-IgE syndrome. Nature 448(7157):1058-1062

Mori T, Miyamoto T, Yoshida H, Asakawa M, Kawasumi M, Kobayashi T, Morioka H, Chiba K, Toyama Y, Yoshimura A (2011) IL-1 $\beta$ and TNF $\alpha$-initiated IL-6-STAT3 pathway is critical in mediating inflammatory cytokines and RANKL expression in inflammatory arthritis. Int Immunol 23(11):701-712

Palomares O, Yaman G, Azkur AK, Akkoc T, Akdis M, Akdis CA (2010) Role of Treg in immune regulation of allergic diseases. Eur J Immunol 40(5):1232-1240

Paul B, Mishra V, Chaudhury B, Awasthi A, Das AB, Saxena U, Saxena A, Chauhan LK, Kumar P, Raisuddin S (2009) Status of Stat3 in an ovalbumin-induced mouse model of asthma: analysis of the role of Socs3 and IL-6. Int Arch Allergy Immunol 148(2):99-108

Saleh A, Shan L, Halayko AJ, Kung S, Gounni AS (2009) Critical role for STAT3 in IL-17A-mediated CCL11 expression in human airway smooth muscle cells. J Immunol 182(6):3357-3365

Sun J, Shi YH, Le GW, Ma XY (2005) Distinct immune response induced by peptidoglycan derived from Lactobacillus sp. World J Gastroenterol 11(40):6330-6337

Wang XQ, Hu G, Kou W, Shen Y, Kang HY, Hong SL (2012) Reciprocal roles of STAT3 and STAT5 in nasal polyposis. Am J Otolaryngol 33(6):741-752

Wei L, Laurence A, O'Shea JJ (2008) New insights into the roles of Stat5a/b and Stat3 in T cell development and differentiation. Semin Cell Dev Biol 19(4):394 400

Xie JJ, Wang J, Tang TT, Chen J, Gao XL, Yuan J, Zhou ZH, Liao MY, Yao R, Yu X, Wang D, Cheng Y, Liao YH, Cheng X (2010) The Th17/Treg functional imbalance during atherogenesis in ApoE(-/-)mice. Cytokine 49(2): 185-193

Yang XO, Panopoulos AD, Nurieva R, Chang SH, Wang D, Watowich SS, Dong C (2007) STAT3 regulates cytokine-mediated generation of inflammatory helper T cells. J Biol Chem 282(13):9358-363

Yang XP, Ghoreschi K, Steward-Tharp SM, Rodriguez-Canales J, Zhu J, Grainger JR, Hirahara K, Sun HW, Wei L, Vahedi G, Kanno Y, O'Shea JJ, Laurence A (2011) Opposing regulation of the locus encoding IL17 through direct, reciprocal actions of STAT3 and STAT5. Nat Immunol 12(3):247-254

Yao Z, Cui Y, Watford WT, Bream JH, Yamaoka K, Hissong BD, Li D, Durum SK, Jiang Q, Bhandoola A, Hennighausen L, O'Shea JJ (2006) Stat5a/b are essential for normal lymphoid development and differentiation. Proc Natl Acad Sci 103(4):1000-1005

Zhao Y, Yang J, Gao YD, Guo W (2010) Th17 immunity in patients with allergic asthma. Int Arch Allergy Immunol 151(4):297-307 